\title{
Higher circulatory HMGB1 accelerates postoperative acute exacerbation of interstitial lung disease in lung cancer
}

\author{
Kakuhiro Yamaguchi ( $\sim$ yamaguchikakuhiro@gmail.com ) \\ Hiroshima University https://orcid.org/0000-0002-9117-1934
}

\section{Satoshi Nakao}

Hiroshima Daigaku Igakubu Daigakuin Ishiyaku Hokengaku Kenkyuka

Hiroshi Iwamoto

Hiroshima Daigaku Igakubu Daigakuin Ishiyaku Hokengaku Kenkyuka

\section{Atsushi Kagimoto}

Hiroshima Daigaku Igakubu Daigakuin Ishiyaku Hokengaku Kenkyuka

\section{Yoshinori Handa}

Hiroshima Daigaku Igakubu Daigakuin Ishiyaku Hokengaku Kenkyuka

\section{Shinjiro Sakamoto}

Hiroshima Daigaku Igakubu Daigakuin Ishiyaku Hokengaku Kenkyuka

\section{Yasushi Horimasu}

Hiroshima Daigaku Igakubu Daigakuin Ishiyaku Hokengaku Kenkyuka

\section{Takeshi Masuda}

Hiroshima Daigaku Igakubu Daigakuin Ishiyaku Hokengaku Kenkyuka

\section{Takahiro Mimae}

Hiroshima Daigaku Igakubu Daigakuin Ishiyaku Hokengaku Kenkyuka

\section{Shintaro Miyamoto}

Hiroshima Daigaku Igakubu Daigakuin Ishiyaku Hokengaku Kenkyuka

\section{Taku Nakashima}

Hiroshima Daigaku Igakubu Daigakuin Ishiyaku Hokengaku Kenkyuka

\section{Yasuhiro Tsutani}

Hiroshima Daigaku Igakubu Daigakuin Ishiyaku Hokengaku Kenkyuka

\section{Kazunori Fujitaka}

Hiroshima Daigaku Igakubu Daigakuin Ishiyaku Hokengaku Kenkyuka

\section{Yoshihiro Miyata}

Hiroshima Daigaku Igakubu Daigakuin Ishiyaku Hokengaku Kenkyuka

\section{Hironobu Hamada}

Hiroshima Daigaku Igakubu Daigakuin Ishiyaku Hokengaku Kenkyuka

\section{Morihito Okada}


Hiroshima Daigaku Igakubu Daigakuin Ishiyaku Hokengaku Kenkyuka

\section{Noboru Hattori}

Hiroshima Daigaku Igakubu Daigakuin Ishiyaku Hokengaku Kenkyuka

\section{Research}

Keywords: acute exacerbation, biomarker, high-mobility group box 1, interstitial lung disease, lung cancer, surgery

Posted Date: August 3rd, 2020

DOl: https://doi.org/10.21203/rs.3.rs-50646/v1

License: (c) (i) This work is licensed under a Creative Commons Attribution 4.0 International License. Read Full License 


\section{Abstract}

Background: Postoperative acute exacerbation of interstitial lung disease (AE-ILD) is a fatal complication in patients with lung cancer and ILD, and it needs to be overcome to improve the long-term outcomes of these patients. However, the molecular target for predicting and preventing this fatal complication remains unclear. High-mobility group box 1 (HMGB1), which is reported to increase due to surgical procedure, activates a pro-inflammatory response associated with acute lung injury. This study aimed to elucidate the association between postoperative AE-ILD and circulatory HMGB1, especially focusing on its predictive potential.

Methods: This study included 152 patients with lung cancer and ILD, who underwent radical surgery between January 2011 and August 2019. We measured HMGB1 serum levels, and investigated the factors affecting HMGB1 and the predictive potential of HMGB1 for postoperative AE-ILD.

Results: Postoperative AE-ILD was developed in 17 (11.2\%) of 152 patients with lung cancer and ILD. HMGB1 serum levels in patients with AE-ILD were significantly higher than those in patients without (median [IQR]: 5.39 [3.29-11.70] ng/mL vs 3.55 [2.07-5.62] ng/mL). Logistic regression analysis revealed that HMGB1 higher levels and longer operative time was independently associated with a higher incidence of postoperative AE-ILD. Furthermore, when HMGB1 and operative time were incorporated into previously reported risk scoring system, the concordance index was 0.876 which is statistically higher than 0.715 calculated by reported scoring system only.

Conclusions: Baseline levels of serum HMGB1 could be a promising biomarker for predicting postoperative AE-ILD, especially when combined with operative time. HMGB1 may be a molecular target of this fatal complication to be overcome.

\section{Background}

High-mobility group box 1 (HMGB1) is a nuclear protein that was characterized to be involved in DNA organization and the regulation of transcription [1]. HMGB1 is released passively during cellular necrosis by almost all cells. It is also secreted actively by immune cells, including monocytes, macrophages, and dendritic cells [2]. Circulatory HMGB1 binds to pattern recognition receptors on the cell surface, such as the receptor for advanced glycation end products (RAGE) and toll-like receptor (TLR). Their interactions result in activating pro-inflammatory response associated with acute lung injury [3, 4]. We have previously reported that higher levels of circulatory HMGB1 at the baseline are associated with the earlier development of acute exacerbation of idiopathic pulmonary fibrosis (AE-IPF) [5]. Additionally, when AEIPF is developed, HMGB1 levels are significantly elevated compared to IPF patients without $A E[5,6]$. Although the trigger of AE-IPF is not sufficiently elucidated, these data suggest that not only higher baseline levels of HMGB1 but also its additional elevation may trigger lung injury superimposed on interstitial lung disease (ILD). 
Postoperative AE-ILD is triggered by surgical procedure [7]. Previous reports have shown that postoperative AE-ILD occurs in $9.3 \%$ of patients, with a mortality rate of $43.9 \%$, in patients with lung cancer and ILD [8]. The pathophysiology of this fatal adverse event is still unclear. However, several studies have demonstrated that HMGB1-induced lung injury is accelerated during surgery. For example, mechanical ventilation induces elevated expression of HMGB1 in lung tissue and bronchoalveolar lavage fluid (BALF), and anti-HMGB1 antibody can cancel the ventilation-induced lung injury $[9,10]$. These observations let us speculate that surgery-induced secretion of HMGB1 would trigger postoperative AEILD, especially in patients with higher levels of HMGB1. Thus, baseline levels of circulatory HMGB1 can be a predictive blood marker of this severe complication.

This study aimed to elucidate the association between serum levels of HMGB1 and the development of postoperative AE-ILD in patients with lung cancer and ILD. First, baseline levels of serum HMGB1 were compared between the patients with and without postoperative AE-ILD. Second, the logistic regression model was used to identify the independent risks of this severe complication. Third, the difference in the incidence of postoperative AE-ILD based on the identified risks was analyzed, especially focusing on HMGB1 and factors associated with surgical procedure. Last, the utility of HMGB1 was evaluated when it combined with already reported risk scoring system using a surgical procedure, male sex, serum levels of Krebs von den Lungen-6 (KL-6), history of AE, preoperative use of steroids, radiological ILD pattern, and vital capacity (VC).

\section{Methods}

\section{Study design and population}

This cohort study consecutively included lung cancer patients with ILD who prospectively collected blood samples before surgery and underwent radical surgery for lung cancer between January 2011 and August 2019 at the Hiroshima University Hospital. The staging of lung cancer was according to the TNM Classification of Malignant Tumors, 8th edition [11]. This study was approved by the Ethics Committee of Hiroshima University Hospital (Gen-38 and E-1707). All participants provided written informed consent for obtaining the blood sample before surgery, and an opt-out method was performed to measure HMGB1 serum levels by using the already gathered samples.

\section{Diagnostic criteria for ILD and postoperative AE-ILD}

The diagnostic criteria for ILD were the existence of bilateral reticulation and consolidation or groundglass attenuation on computed tomography (CT). The CT pattern was classified by using the American Thoracic Society, European Respiratory Society, Japanese Respiratory Society, and Latin American Thoracic Association (ATS/ERS/JRS/ALAT) clinical practice guideline of IPF; it was classified as usual interstitial pneumonia (UIP) pattern, possible UIP pattern, and inconsistent with UIP pattern [12].

Postoperative AE-ILD caused by lung resection was defined based on the criteria proposed by previous studies for the disease and the ATS/ERS/JRS/ALAT statement as follows: (i) acute worsening or 
development of dyspnea and oxygen desaturation; (ii) CT showing new ground-glass abnormality bilaterally and/or consolidation; (iii) no evidence of other alternative causes, such as pulmonary infection, cardiac failure, pulmonary embolism, and pneumothorax; and (iv) development of AE-ILD within 30 days after lung resection [12-15].

\section{Measurement of serum HMGB1 concentrations}

Serum samples were collected before surgery at our hospital and stored at $-80^{\circ} \mathrm{C}$. Serum levels of HMGB1 were measured using commercially available enzyme-linked immunosorbent assay kits according to the manufacturer's instructions (HMGB1 ELISA Kit II [Shino-Test Corporation, Tokyo]).

\section{Statistical analysis}

Values were expressed as mean \pm standard deviation or median (interquartile range [IQR]) according to their distribution. Normally and non-normally distributed variables were evaluated using the t-test and Mann-Whitney U tests, respectively. Pearson's chi-squared tests were also conducted. Bonferroni correction was applied for multiple comparisons. Linear regression analysis was conducted to study the independent effects of age, sex, smoking history, lung function parameters, ILD pattern, serum levels of $\mathrm{KL}-6$, preoperative steroid use, and history of $\mathrm{AE}$; on serum HMGB1 levels. Univariate and multivariate logistic regression analyses were conducted to identify independent risk factors for postoperative AE-ILD development and to estimate the respective odds ratios and their $95 \%$ confidence intervals $(\mathrm{Cl})$. Confounders in the multivariate analysis were selected by stepwise method from all factors in univariate analysis. The receiver operating characteristic (ROC) curve analysis was performed to identify the optimal cut-off levels of serum HMGB1 and operative time for predicting the development of postoperative AEILD. C-statistics were performed to evaluate and compare logistic models that analyzed the predictive accuracy for postoperative AE-ILD. All statistical analyses were performed using JMP version 14.1.0 (SAS Institute Inc., Cary, NC, USA). A $P$-value of less than 0.05 was considered statistically significant.

\section{Results}

\section{Patient characteristics}

The subjects were 157 patients with lung cancer and ILD who underwent radical surgery and had blood samples collected before surgery. After excluding 5 patients who had another primary cancer or active autoimmune disease, 152 patients with lung cancer and ILD were included in the analysis (Table 1). 
Table 1

Baseline characteristics

\begin{tabular}{|c|c|c|c|c|}
\hline & All subjects & AE-ILD (+) & AE-ILD (-) & $\begin{array}{l}P \\
\text { value* }\end{array}$ \\
\hline Subjects, n (\%) & 152 & $17(11.2)$ & $135(88.8)$ & \\
\hline Age, years & $72.9 \pm 7.5$ & $71.7 \pm 7.5$ & $73.1 \pm 7.5$ & 0.487 \\
\hline Sex, male/female & $126 / 26$ & $17 / 0$ & $109 / 26$ & 0.045 \\
\hline $\begin{array}{l}\text { Smoking history, pack- } \\
\text { years }\end{array}$ & $50.0(30.0-67.5)$ & $60.0(39.0-95.0)$ & $47.5(30.0-65.6)$ & 0.211 \\
\hline VC, \% predicted & $92.4(80.8-101.6)$ & $79.3(73.9-92.6)$ & $93.2(83.8-101.8)$ & 0.014 \\
\hline DLco, \% predicted & $56.5 \pm 17.4$ & $51.2 \pm 21.1$ & $57.2 \pm 16.8$ & 0.237 \\
\hline ILD pattern & & & & 0.267 \\
\hline UIP & 52 & 9 & 43 & \\
\hline Possible UIP & 67 & 5 & 62 & \\
\hline Inconsistent with UIP & 33 & 3 & 30 & \\
\hline $\mathrm{KL}-6, \mathrm{U} / \mathrm{mL}$ & $\begin{array}{l}432.0 \\
(284.7-700.0)\end{array}$ & $\begin{array}{l}670.0 \\
(360.0-1139.0)\end{array}$ & $\begin{array}{l}421.0 \\
(283.0-669.0)\end{array}$ & 0.028 \\
\hline $\begin{array}{l}\text { Preoperative steroid use, } \\
+/-\end{array}$ & $15 / 137$ & $2 / 15$ & $13 / 122$ & 0.676 \\
\hline History of $A E,+/-$ & $9 / 143$ & $3 / 14$ & $6 / 129$ & 0.064 \\
\hline Histology & & & & 0.689 \\
\hline Adenocarcinoma & 68 & 6 & 62 & \\
\hline $\begin{array}{l}\text { Squamous cell } \\
\text { carcinoma }\end{array}$ & 56 & 7 & 49 & \\
\hline Small cell carcinoma & 11 & 2 & 9 & \\
\hline Others & 17 & 2 & 15 & \\
\hline cStage, $\nabla / \otimes / \otimes A / \otimes B$ & $122 / 21 / 7 / 2$ & $13 / 3 / 0 / 1$ & $109 / 18 / 7 / 1$ & 0.300 \\
\hline
\end{tabular}

*All $P$-values are evaluated by comparing between patients with and without postoperative AE-ILD using the t-test and Mann-Whitney U tests for normally and non-normally distributed variables and using Pearson's chi-squared test.

Data are presented as mean \pm standard deviation or median (interquartile range) according to their distribution.

AE-ILD, acute exacerbation of interstitial lung disease; DLco, diffusing capacity for carbon monoxide; ILD, interstitial lung disease; KL-6, Krebs von den Lungen-6; UIP, usual interstitial pneumonia; VC, vital capacity; 


\begin{tabular}{|c|c|c|c|c|}
\hline & All subjects & AE-ILD (+) & AE-ILD (-) & $\begin{array}{l}P \\
\text { value* }\end{array}$ \\
\hline \multicolumn{4}{|l|}{ Surgical procedure } & \multirow[t]{4}{*}{0.838} \\
\hline Wedge resection & 41 & 4 & 37 & \\
\hline Segmentectomy & 33 & 3 & 30 & \\
\hline Lobectomy & 78 & 10 & 68 & \\
\hline Operative time, $\min$ & $149(108-196)$ & $200(138-252)$ & $146(106-181)$ & 0.022 \\
\hline Bleeding volume, $\mathrm{mL}$ & $66(24-110)$ & $155(73-301)$ & $60(23-100)$ & 0.003 \\
\hline \multicolumn{5}{|c|}{$\begin{array}{l}\text { *All } P \text {-values are evaluated by comparing between patients with and without postoperative AE-ILD } \\
\text { using the t-test and Mann-Whitney U tests for normally and non-normally distributed variables and } \\
\text { using Pearson's chi-squared test. }\end{array}$} \\
\hline \multicolumn{5}{|c|}{$\begin{array}{l}\text { Data are presented as mean } \pm \text { standard deviation or median (interquartile range) according to their } \\
\text { distribution. }\end{array}$} \\
\hline \multicolumn{5}{|c|}{$\begin{array}{l}\text { AE-ILD, acute exacerbation of interstitial lung disease; DLco, diffusing capacity for carbon monoxide; } \\
\text { ILD, interstitial lung disease; KL-6, Krebs von den Lungen-6; UIP, usual interstitial pneumonia; VC, vital } \\
\text { capacity; }\end{array}$} \\
\hline
\end{tabular}

The patients with postoperative AE-ILD were male dominant and had significantly lower VC (\% predicted), higher levels of serum KL-6, longer operative time, and higher bleeding volume than the patients without postoperative AE-ILD (Table 1). There was a significant correlation between operative time and bleeding volume $\left(r_{s}=0.640, P<0.001\right)$.

\section{Baseline concentration of HMGB1 and its predictive potential for postoperative AE-ILD}

Overall, 17 patients developed postoperative AE-ILD. Serum levels of HMGB1 were significantly higher in patients with postoperative AE-ILD than in those without (median [IQR]: 5.39 [3.29-11.70] ng/mL vs 3.55 [2.07-5.62] ng/mL, $P=0.031$ ) (Fig. 1). Higher levels of HMGB1 were significantly and independently associated with younger age, higher pack-year smoking history, and lower VC (Table 2). Univariate logistic regression analysis revealed that lower $\mathrm{VC}$, history of $A E$, higher levels of serum $K L-6$, longer operative time, higher bleeding volume, and higher levels of HMGB1 were significantly associated with the development of postoperative AE-ILD. Multivariate stepwise analysis revealed that lower VC, history of AE, higher levels of serum KL-6, longer operative time, and higher levels of HMGB1 independently elevated the risk of this fatal complication (Table 3). 
Table 2

Linear regression analysis to elucidate the factors associated with HMGB1 serum levels

\begin{tabular}{|c|c|c|c|}
\hline Variables & $\beta$ & $t$ & $P$-value \\
\hline \multicolumn{4}{|l|}{ Univariate analysis } \\
\hline Age, years & -0.165 & -2.04 & $0.043^{*}$ \\
\hline Sex, male & 0.127 & 1.57 & 0.118 \\
\hline Smoking history, pack-years & 0.243 & 3.06 & $0.003^{*}$ \\
\hline VC, \%predicted & -0.179 & -2.23 & $0.027 *$ \\
\hline DLco, \% predicted & -0.099 & -1.22 & 0.226 \\
\hline ILD pattern, UIP + possible UIP & 0.044 & 0.54 & 0.593 \\
\hline $\mathrm{KL}-6, \mathrm{U} / \mathrm{mL}$ & 0.013 & 0.16 & 0.876 \\
\hline Preoperative steroid use, + & -0.008 & -0.10 & 0.917 \\
\hline History of $A E,+$ & 0.169 & 2.10 & $0.037^{*}$ \\
\hline \multicolumn{4}{|l|}{ Multivariate analysis } \\
\hline Age, years & -0.170 & -2.19 & $0.030 *$ \\
\hline Smoking history, pack-years & 0.210 & 2.62 & $0.010^{\star}$ \\
\hline VC, \% predicted & -0.164 & -2.08 & $0.040 *$ \\
\hline History of $\mathrm{AE},+$ & 0.088 & 1.08 & 0.284 \\
\hline \multicolumn{4}{|l|}{ * $P<0.05$ Linear regression model } \\
\hline $\begin{array}{l}\text { AE-ILD, acute exacerbation; DLco, diff } \\
\text { group box } 1 \text {; ILD, interstitial lung disea } \\
\text { pneumonia; VC, vital capacity; }\end{array}$ & $\begin{array}{l}\text { bon mo } \\
\text { en Lung }\end{array}$ & $\begin{array}{l}\text { HMGB } \\
\text { IP, usua }\end{array}$ & $\begin{array}{l}\text { h-mobility } \\
\text { stitial }\end{array}$ \\
\hline
\end{tabular}


Table 3

Logistic regression analysis elucidating the risk of postoperative AE-ILD

Variables

Univariate analysis

Age, years

Sex, male

pack-year

VC, \% predicted

DLco, \% predicted

NSCLC vs SCLC

Stage I vs II-III

History of AE-ILD, +

ILD pattern, UIP + possible UIP

$\mathrm{KL}-6, \mathrm{U} / \mathrm{mL}$

Preoperative steroid use, +

Surgical procedure, Segmentectomy/lobectomy

Operative time, $\min$

Bleeding volume, $\mathrm{mL}$

HMGB1, ng/mL

Multivariate stepwise analysis"

VC, \% predicted

History of $A E,+$

$\mathrm{KL}-6, \mathrm{U} / \mathrm{mL}$

Preoperative steroid use, +
Odds

$95 \% \mathrm{Cl}$

P-value
$0.975 \quad 0.911-1.045 \quad 0.475$

Not calculated

$\begin{array}{lll}1.010 & 0.999-1.020 & 0.076\end{array}$

$\begin{array}{lll}0.965 & 0.936-0.994 & 0.020^{*}\end{array}$

$\begin{array}{lll}0.979 & 0.950-1.010 & 0.180\end{array}$

$\begin{array}{lll}0.536 & 0.106-2.715 & 0.451\end{array}$

$\begin{array}{lll}0.775 & 0.233-2.573 & 0.677\end{array}$

$4.607 \quad 1.037-20.475 \quad 0.045^{*}$

$\begin{array}{lll}1.221 & 0.328-4.546 & 0.766\end{array}$

$1.001 \quad 1.000-1.001 \quad 0.043^{*}$

$\begin{array}{lll}1.251 & 0.257-6.089 & 0.781\end{array}$

$1.227 \quad 0.376-4.000 \quad 0.735$

$1.010 \quad 1.003-1.018 \quad 0.008^{*}$

$1.003 \quad 1.001-1.005 \quad 0.008^{*}$

$1.141 \quad 1.050-1.239 \quad 0.002^{*}$

* $P<0.05$ Logistic regression analysis

\# Confounders in the multivariate analysis were selected by stepwise method from all factors in univariate analysis.

AE-ILD, acute exacerbation of interstitial lung disease; $\mathrm{Cl}$, confidence interval; $\mathrm{DLCO}$, diffusing capacity for carbon monoxide; HMGB1, high mobility group box 1; ILD, interstitial lung disease; KL-6, Krebs von den Lungen-6; NSCLC, non-small cell lung cancer; SCLC, small cell lung cancer; UIP, usual interstitial pneumonia; $\mathrm{VC}$, vital capacity; 


\begin{tabular}{|lccc|}
\hline Variables & Odds & $95 \%$ Cl & $P$-value \\
\hline Operative time, min & 1.016 & $1.004-1.028$ & $0.011^{*}$ \\
\hline Bleeding volume, $\mathrm{mL}$ & 1.002 & $0.999-1.004$ & 0.161 \\
\hline HMGB1, $\mathrm{ng} / \mathrm{mL}$ & 1.132 & $1.008-1.271$ & $0.036^{*}$ \\
\hline * $P<0.05$ Logistic regression analysis & & & \\
\hline $\begin{array}{l}\text { \# Confounders in the multivariate analysis were selected by stepwise method from all factors in } \\
\text { univariate analysis. }\end{array}$ & & \\
\hline $\begin{array}{l}\text { AE-ILD, acute exacerbation of interstitial lung disease; Cl, confidence interval; DLco, diffusing capacity } \\
\text { for carbon monoxide; HMGB1, high mobility group box 1; ILD, interstitial lung disease; KL-6, Krebs von } \\
\text { den Lungen-6; NSCLC, non-small cell lung cancer; SCLC, small cell lung cancer; UIP, usual interstitial } \\
\text { pneumonia; VC, vital capacity; }\end{array}$ \\
\hline
\end{tabular}

\section{The risk stratification based on HMGB1 and operative time}

To elucidate the influence of the surgical procedure as the trigger of postoperative AE-ILD, the incidence was evaluated focusing on HMGB1 baseline levels and operative time identified as the independent risk factor of the disease in this study. ROC curve analysis revealed that the optimal cut-off level of serum HMGB1 and operative time for predicting postoperative AE-ILD was $3.82 \mathrm{ng} / \mathrm{mL}$ (AUC 0.661, specificity $51.9 \%$, and sensitivity $76.5 \%$ ) and 200 min (AUC 0.671 , specificity $58.9 \%$, and sensitivity $80.0 \%$ ). Each of HMGB1 higher than $3.82 \mathrm{ng} / \mathrm{mL}$ and operative time longer than 200 min was associated with a higher incidence of postoperative AE-ILD (Fig. 2a, 2b). Additionally, the patients with both HMGB1 higher than $3.82 \mathrm{ng} / \mathrm{mL}$ and operative time longer than $200 \mathrm{~min}$ had the highest risk of the disease compared to those with either HMGB1-high or operative time-long and those with neither (Fig. 2c).

\section{Comparisons of C-statistics in predictive models for postoperative AE-ILD}

The C-statistics showed that HMGB1 alone had modest predictive accuracy for postoperative AE-ILD (concordance index $[c-i n d e x]=0.661)$ (Supplementary Table S1). However, when numerical variables of HMGB1 were combined with covariates calculated by previously reported risk scoring system, i.e., $5 \times$ (history of $\mathrm{AE})+4 \times$ (surgical procedures) $+4 \times(\mathrm{UIP}$ appearance in $\mathrm{CT}$ scan $)+3 \times$ (male sex $)+3 \times$ (preoperative steroid use) $+2 \times$ (serum KL-6 level higher than $1000 \mathrm{U} / \mathrm{mL})+1 \times($ vital capacity lower than $80 \%)$ [14], the c-index tended to increase to $0.799(P=0.109)$, which was higher than 0.715 calculated by covariates only (Fig. 3). Additionally, when numerical variables of HMGB1 and operative time were incorporated into a model with covariates, the c-index was 0.876 , which is statistically and significantly higher than that calculated by covariates only (Fig. 3).

\section{Discussion}


Postoperative AE-ILD is a fatal complication, and thus it is important to increase its predictive accuracy before surgery. This study showed that baseline levels of serum HMGB1 in patients with postoperative AE-ILD were significantly higher than those without AE-ILD. Additionally, higher levels of HMGB1 were significantly and independently associated with a higher incidence of this fatal complication. Lastly, HMGB1 had the potential to improve the predictive accuracy of risk scoring system, which had been already reported by Sato et al $[8,14]$. These data indicate that HMGB1 can be a promising biomarker to predict the postoperative $A E$ in patients with lung cancer and ILD.

This study firstly showed that higher levels of HMGB1 were significantly associated with a higher incidence of postoperative AE-ILD in patients with lung cancer and ILD. We and others previously reported that HMGB1 levels in serum and BALF in patients with IPF were significantly elevated than those in healthy subjects $[5,16]$. Additionally, we previously reported that higher levels of HMGB1 were associated with earlier onset of AE in patients with IPF [5]. HMGB1 itself accelerates pro-inflammatory signaling via interacting with RAGE and TLR4 [3, 4], and intratracheal administration of HMGB1 induces acute lung injury [17]. At the same time, some reports showed that injured alveolar epithelial cells highly expressed HMGB1 [16], indicating that one of the HMGB1 sources in patients with ILD is injured lung tissue. This study also showed that there was a negative correlation between HMGB1 and VC. These data suggest that higher levels of HMGB1 reflect not only pro-inflammatory conditions in the lung but also pre-existing lung damage. Thus, its higher levels can predict the development of postoperative AE-ILD.

This study showed that the patients, both with higher HMGB1 and longer operative time, had a significantly higher risk of this fatal complication than patients with either higher HMGB1 or longer operative time and patients with neither. When operative time becomes longer, it requires a longer time of artificial ventilation, which increases the expression of HMGB1 in BALF and lung tissue in a tidal volumeand time-dependent manner $[9,10]$. Exposure to hyperoxia during artificial ventilation also promotes increasing expression of HMGB1 in a time-dependent manner [18]. This mechanical ventilation- and hyperoxia-induced lung injury is canceled by inhibition of HMGB1 $[9,18]$. Additionally, higher bleeding volume, which was correlated with operative time and showed potential association with postoperative AE-ILD in this study, is also reported to increase the expression of HMGB1 in plasma and lung [19]. These data indicate that surgery accelerates inflammatory response via additional HMGB1 secretion, which results in the development of postoperative AE-ILD, especially in patients with HMGB1 higher levels.

This study also showed that HMGB1, especially when combined with operative time, could improve the predictive accuracy of postoperative AE-ILD based on previously reported risk scoring systems in patients with lung cancer and ILD [14]. Surgical resection is generally performed as a standard radical treatment for lung cancer. However, it is associated with higher postoperative morbidity of complications when the patient has concurrent ILD [20]. Preventing this fatal complication is crucial to improve the long-term outcome in patients with lung cancer and ILD who underwent surgical resection. One potential preventive strategy is that patients with higher HMGB1 accompanied with reported risks would be treated with wedge resection, because it generally needs shorter operative time and implies less bleeding volume than segmentectomy/lobectomy. Another potential strategy is to use HMGB1-inhibitory drugs. Some existing

Page $11 / 18$ 
drugs are reported to inhibit HMGB1-induced inflammatory signal via decreasing HMGB1 secretion and capturing HMGB1 [21]. Further investigations are needed to elucidate the utility of circulatory HMGB1 as a predictive biomarker and molecular target for preventing postoperative AE-ILD in patients with lung cancer and ILD.

This study has several limitations. It was a single-institution study, and the number of patients with postoperative AE-ILD was relatively small. These may substantially impact the generalizability of the study findings. Multicenter studies with a larger sample size are needed to validate the predictive value and the optimal cut off levels of HMGB1.

\section{Conclusions}

Higher levels of circulatory HMGB1 are associated with postoperative AE-ILD in patients with lung cancer and ILD. The combination of HMGB1 and operative time might improve the predictive accuracy of this fatal complication. These data confirm that HMGB1 is associated with the molecular mechanism of acute lung injury superimposed on ILD by focusing on surgery as a trigger of $A E$.

\section{Abbreviations}

AE, acute exacerbation; ALAT, Latin American Thoracic Association; ATS, American Thoracic Society; BALF, bronchoalveolar lavage fluid; $\mathrm{Cl}$, confidence interval; $\mathrm{CT}$, computed tomography; DLco, diffusing capacity for carbon monoxide; ERS, European Respiratory Society; HMGB1, high mobility group box 1; ILD, interstitial lung disease; IPF, idiopathic pulmonary fibrosis; IQR, interquartile range; JRS, Japanese Respiratory Society; KL-6, Krebs von den Lungen-6; RAGE, receptor for advanced glycation end products; ROC, receiver operating characteristic; TLR, toll-like receptor; UIP, usual interstitial pneumonia; VC, vital capacity;

\section{Declarations}

Ethics approval and consent to participate; This study was approved by the Ethics Committee of Hiroshima University Hospital (Gen-38 and E-1707). All participants provided written informed consent for obtaining the blood sample before surgery, and an opt-out method was performed to measure HMGB1 serum levels by using the already gathered samples.

Consent for publication; Not applicable.

Availability of data and materials; Please contact author for data requests.

Competing interests; The authors declare that they have any competing interests (both financial and nonfinancial) related to this manuscript. 
Funding; This work was supported by Grants-in-Aid for Scientific Research from the Ministry of Education, Culture, Sports, Science and Technology of Japan and by the Diffuse Lung Diseases Research Group from the Ministry of Health, Labour and Welfare, Japan.

Authors' contributions; $\mathrm{KY}$ and $\mathrm{SN}$ designed the study, performed the data analysis and interpretation, and wrote the manuscript. $\mathrm{HI}, \mathrm{SS}, \mathrm{YH}, \mathrm{TM}, \mathrm{SM}, \mathrm{TN}, \mathrm{KF}, \mathrm{HH}$, and $\mathrm{NH}$ designed and interpreted the data. $\mathrm{AK}, \mathrm{YH}$, $\mathrm{TM}, \mathrm{YT}, \mathrm{YM}$, and $\mathrm{MO}$ collected samples and interpreted the data. All authors helped to draft the manuscript and approved the final manuscript.

Acknowledgements; Not applicable

\section{References}

1. Klune JR, Dhupar R, Cardinal J, Billiar TR, Tsung A: HMGB1: endogenous danger signaling. Mol Med 2008, 14:476-484.

2. Ulloa L, Messmer D: High-mobility group box 1 (HMGB1) protein: friend and foe. Cytokine Growth Factor Rev 2006, 17:189-201.

3. Deng Y, Yang Z, Gao Y, Xu H, Zheng B, Jiang M, Xu J, He Z, Wang X: Toll-like receptor 4 mediates acute lung injury induced by high mobility group box-1. PLoS One 2013, 8:e64375.

4. Wu X, Mi Y, Yang H, Hu A, Zhang Q, Shang C: The activation of HMGB1 as a progression factor on inflammation response in normal human bronchial epithelial cells through RAGE/JNK/NF-kappaB pathway. Mol Cell Biochem 2013, 380:249-257.

5. Yamaguchi K, Iwamoto H, Sakamoto S, Horimasu Y, Masuda T, Miyamoto S, Nakashima T, Ohshimo S, Fujitaka K, Hamada H, Hattori N: Serum high-mobility group box 1 is associated with the onset and severity of acute exacerbation of idiopathic pulmonary fibrosis. Respirology 2019.

6. Abe S, Hayashi H, Seo Y, Matsuda K, Kamio K, Saito Y, Usuki J, Azuma A, Kudo S, Gemma A: Reduction in serum high mobility group box-1 level by polymyxin B-immobilized fiber column in patients with idiopathic pulmonary fibrosis with acute exacerbation. Blood Purif 2011, 32:310-316.

7. Amundson WH, Racila E, Allen T, Dincer HE, Tomic R, Bhargava M, Perlman DM, Kim HJ: Acute exacerbation of interstitial lung disease after procedures. Respir Med 2019, 150:30-37.

8. Sato T, Teramukai S, Kondo H, Watanabe A, Ebina M, Kishi K, Fujii Y, Mitsudomi T, Yoshimura M, Maniwa T, et al: Impact and predictors of acute exacerbation of interstitial lung diseases after pulmonary resection for lung cancer. J Thorac Cardiovasc Surg 2014, 147:1604-1611.e1603.

9. Ogawa EN, Ishizaka A, Tasaka S, Koh H, Ueno H, Amaya F, Ebina M, Yamada S, Funakoshi Y, Soejima $\mathrm{J}$, et al: Contribution of high-mobility group box-1 to the development of ventilator-induced lung injury. Am J Respir Crit Care Med 2006, 174:400-407.

10. van Zoelen MA, Ishizaka A, Wolthuls EK, Choi G, van der Poll T, Schultz MJ: Pulmonary levels of highmobility group box 1 during mechanical ventilation and ventilator-associated pneumonia. Shock 2008, 29:441-445. 
11. Goldstraw P, Chansky K, Crowley J, Rami-Porta R, Asamura H, Eberhardt WE, Nicholson AG, Groome P, Mitchell A, Bolejack V: The IASLC Lung Cancer Staging Project: Proposals for Revision of the TNM Stage Groupings in the Forthcoming (Eighth) Edition of the TNM Classification for Lung Cancer. $J$ Thorac Oncol 2016, 11:39-51.

12. Raghu G, Collard HR, Egan JJ, Martinez FJ, Behr J, Brown KK, Colby TV, Cordier JF, Flaherty KR, Lasky JA, et al: An official ATS/ERS/JRS/ALAT statement: idiopathic pulmonary fibrosis: evidence-based guidelines for diagnosis and management. Am J Respir Crit Care Med 2011, 183:788-824.

13. Tsutani Y, Mimura T, Kai Y, Ito M, Misumi K, Miyata Y, Okada M: Outcomes after lobar versus sublobar resection for clinical stage I non-small cell lung cancer in patients with interstitial lung disease. $J$ Thorac Cardiovasc Surg 2017, 154:1089-1096.e1081.

14. Sato T, Kondo H, Watanabe A, Nakajima J, Niwa H, Horio H, Okami J, Okumura N, Sugio K, Teramukai $\mathrm{S}$, et al: A simple risk scoring system for predicting acute exacerbation of interstitial pneumonia after pulmonary resection in lung cancer patients. Gen Thorac Cardiovasc Surg 2015, 63:164-172.

15. Collard HR, Moore BB, Flaherty KR, Brown KK, Kaner RJ, King TE, Jr., Lasky JA, Loyd JE, Noth I, Olman MA, et al: Acute exacerbations of idiopathic pulmonary fibrosis. Am J Respir Crit Care Med 2007, 176:636-643.

16. Hamada N, Maeyama T, Kawaguchi T, Yoshimi M, Fukumoto J, Yamada M, Yamada S, Kuwano K, Nakanishi Y: The role of high mobility group box1 in pulmonary fibrosis. Am J Respir Cell Mol Biol 2008, 39:440-447.

17. Ueno H, Matsuda T, Hashimoto S, Amaya F, Kitamura Y, Tanaka M, Kobayashi A, Maruyama I, Yamada S, Hasegawa N, et al: Contributions of high mobility group box protein in experimental and clinical acute lung injury. Am J Respir Crit Care Med 2004, 170:1310-1316.

18. Entezari M, Javdan M, Antoine DJ, Morrow DM, Sitapara RA, Patel V, Wang M, Sharma L, Gorasiya S, Zur M, et al: Inhibition of extracellular HMGB1 attenuates hyperoxia-induced inflammatory acute lung injury. Redox Biol 2014, 2:314-322.

19. Kim JY, Park JS, Strassheim D, Douglas I, Diaz del Valle F, Asehnoune K, Mitra S, Kwak SH, Yamada S, Maruyama I, et al: HMGB1 contributes to the development of acute lung injury after hemorrhage. Am J Physiol Lung Cell Mol Physiol 2005, 288:L958-965.

20. Chiyo M, Sekine Y, Iwata T, Tatsumi K, Yasufuku K, lyoda A, Otsuji M, Yoshida S, Shibuya K, lizasa T, et al: Impact of interstitial lung disease on surgical morbidity and mortality for lung cancer: analyses of short-term and long-term outcomes. J Thorac Cardiovasc Surg 2003, 126:1141-1146.

21. Venereau E, De Leo F, Mezzapelle R, Careccia G, Musco G, Bianchi ME: HMGB1 as biomarker and drug target. Pharmacol Res 2016, 111:534-544.

\section{Figures}




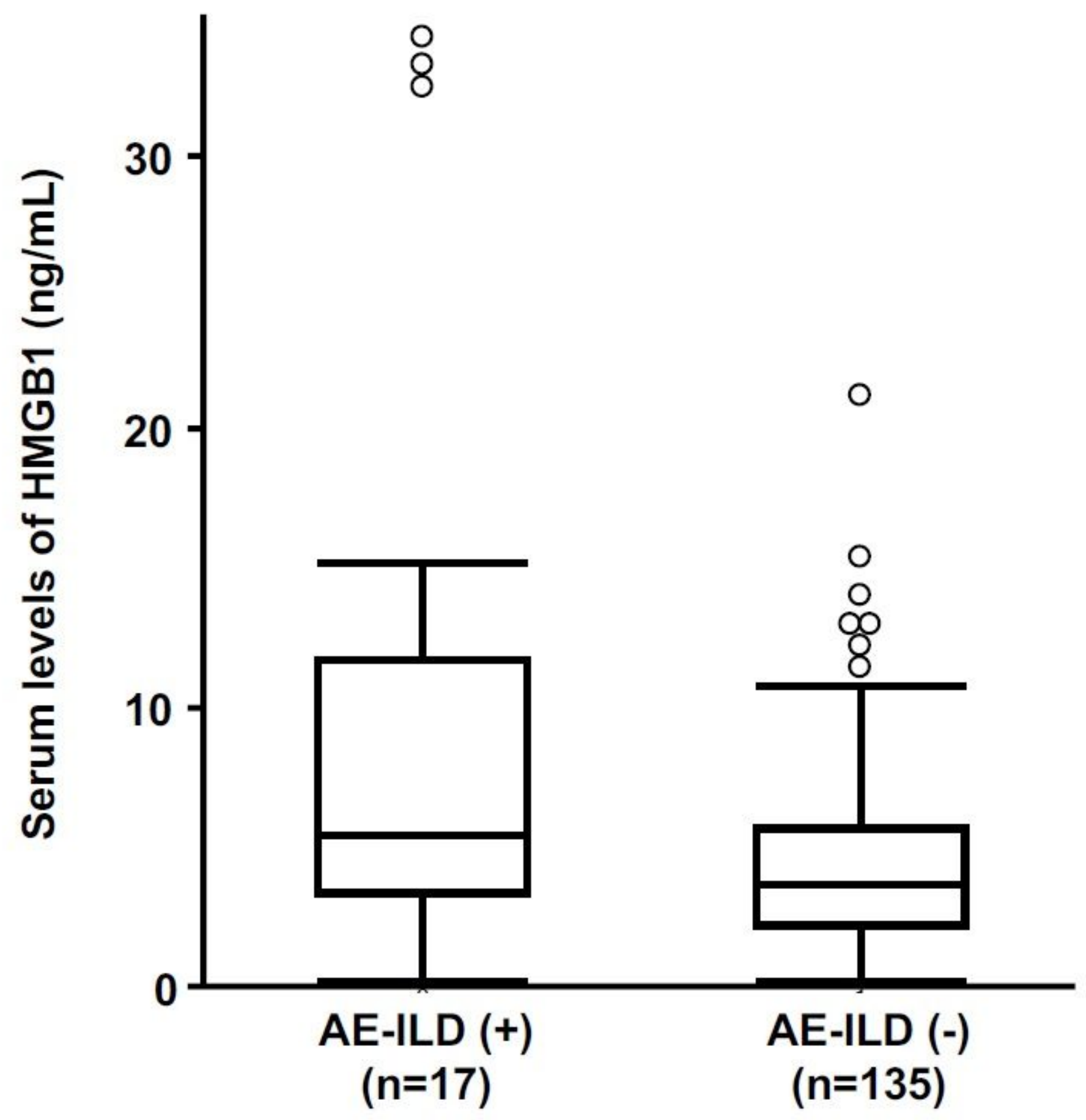

Figure 1

Baseline levels of serum HMGB1 Serum levels of HMGB1 in patients with postoperative acute exacerbation of interstitial lung disease (AE-ILD) were higher than those in patients without (median [IQR]: 5.39 [3.29ه11.70] ng/mL vs. 3.55 [2.07凶5.62] ng/mL, $P=0.031$ ). Boxes represent the 25th to 75th percentiles; solid lines within the boxes show the median values; whiskers represent the 10th and 90th percentiles; the circles represent outliers. ${ }^{*} \mathrm{P}<0.05$ using the Mann-Whitney $\mathrm{U}$ test. IQR, interquartile range 
(a) HMGB1

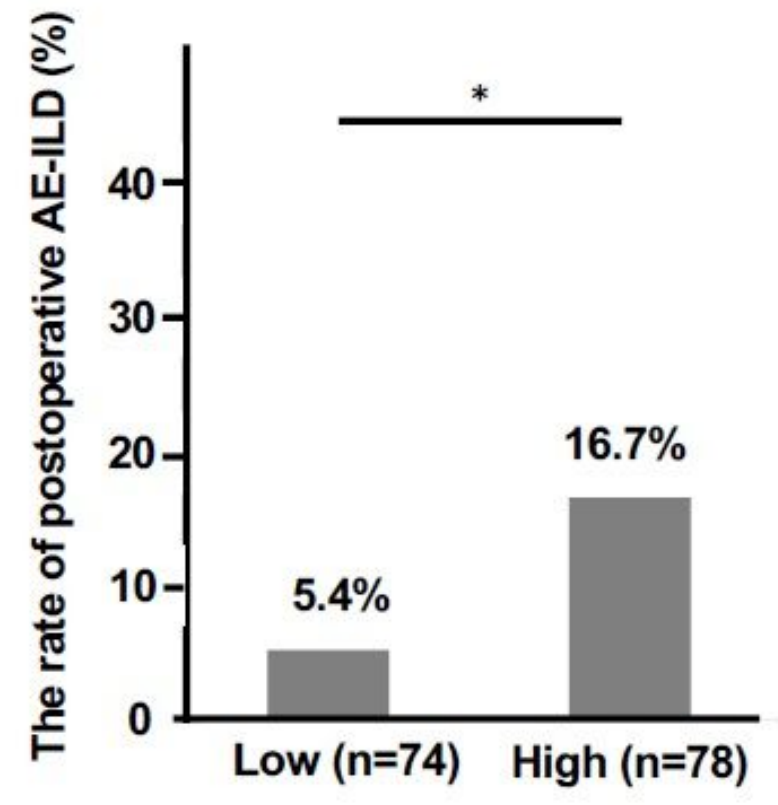

(b) Operative time

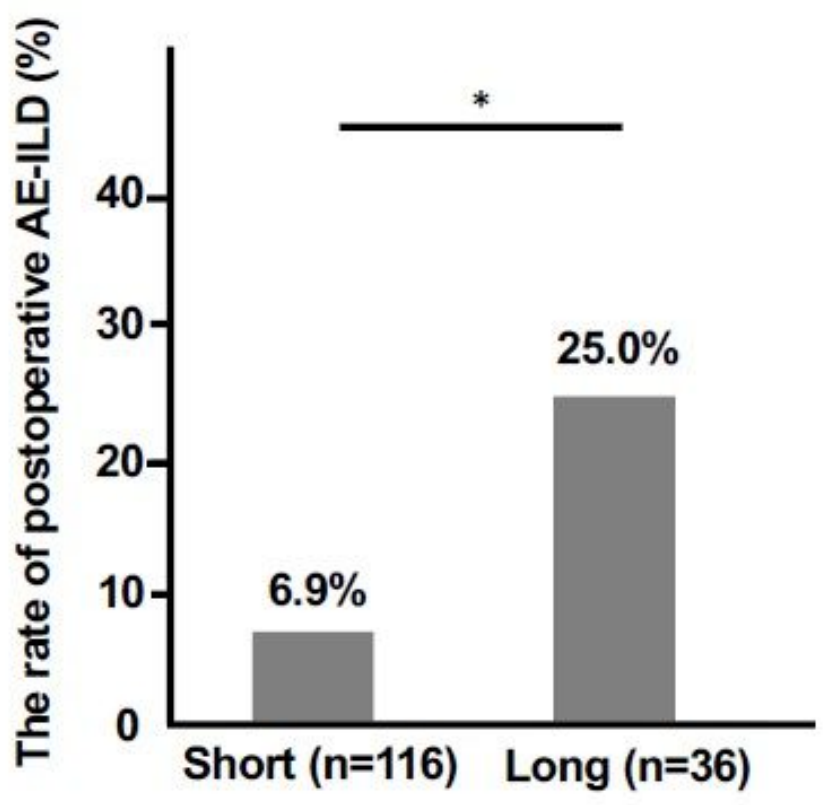

(c) HMGB1 and operative time

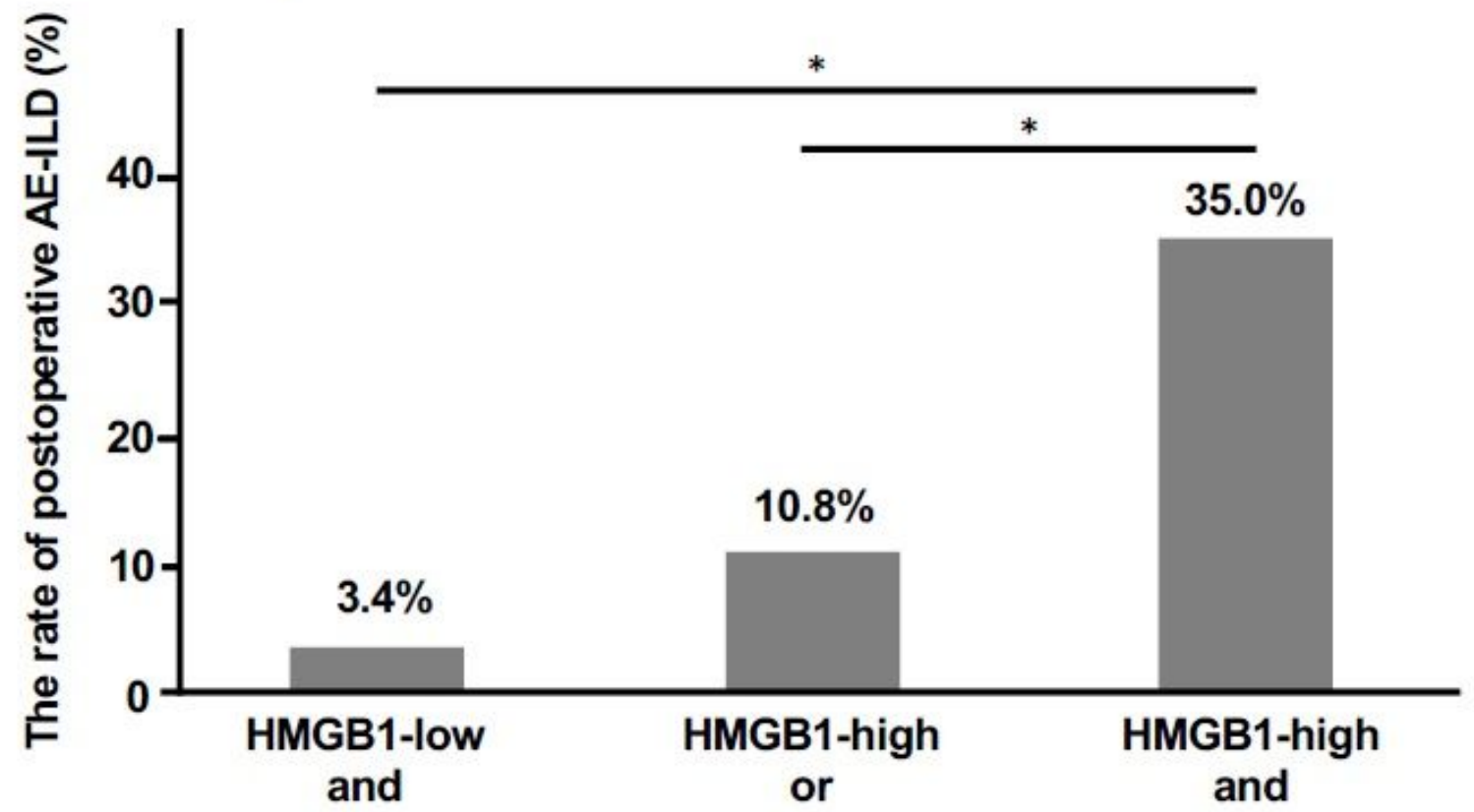

Operative time-short Operative time-long Operative time-long $(n=58)$ $(n=74)$

$(n=20)$

Figure 2

The incidence of postoperative AE-ILD based on HMGB1 and operative time The cut-off levels of HMGB1 and operative time were $3.82 \mathrm{ng} / \mathrm{mL}$ and $200 \mathrm{~min}$, respectively. The incidence of postoperative acute exacerbation of interstitial lung disease (AE-ILD) was significantly higher (a) in patients with HMGB1-high and (b) in those with operative time-long. Additionally, when HMGB1 and operative time were combined, (c) the incidence in patients both with HMGB1-high and operative time-long was statistically the highest 
compared to that in other two groups. * $\mathrm{P}<0.05$ using the Pearson's chi-squared tests with Bonferroni correction.

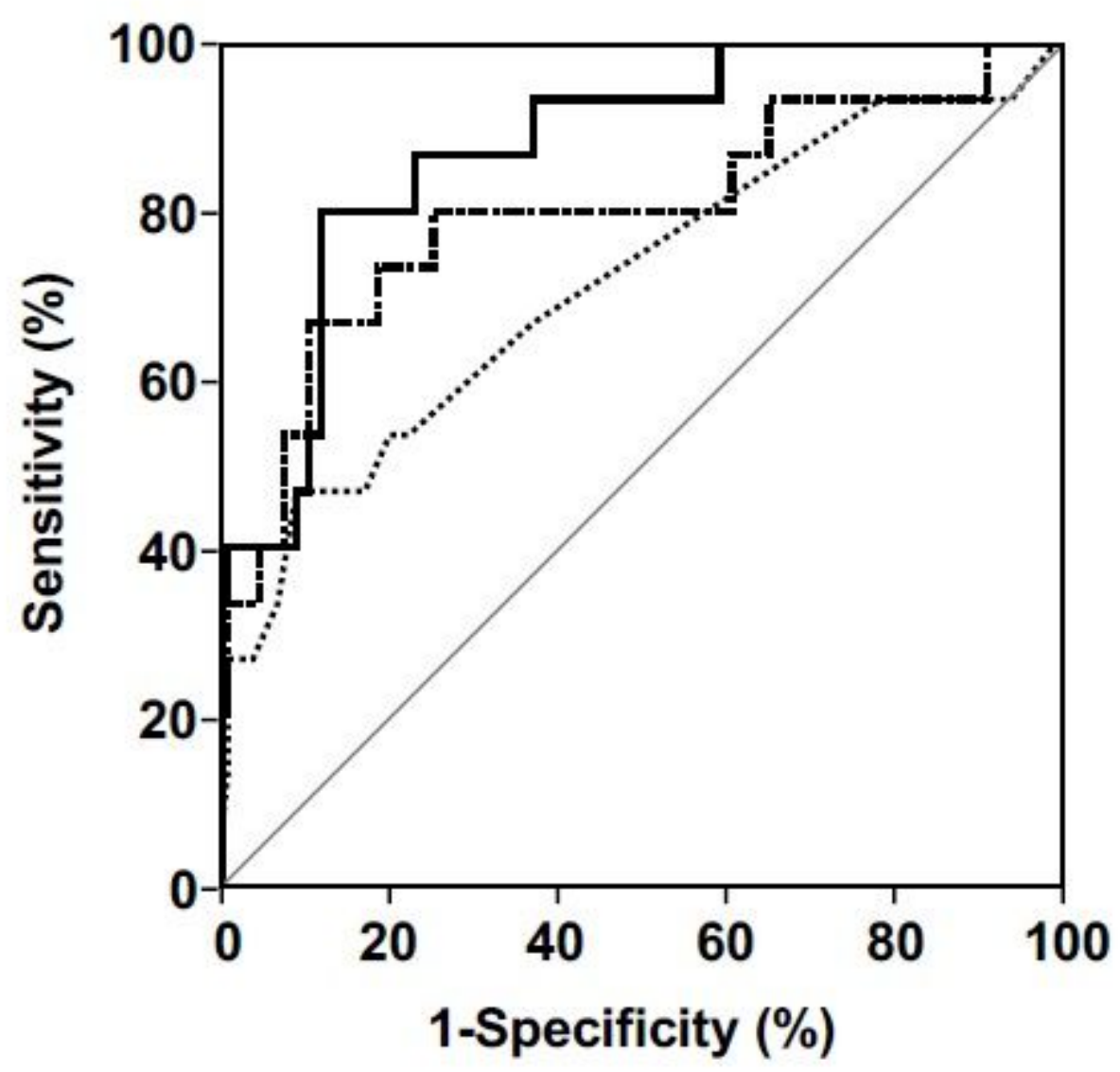

C-index P-value

- Covariates + HMGB1 + operative time $\quad 0.876 \quad 0.044$

-.-- Covariates + HMGB1 $0.799 \quad 0.109$

....... Covariates only $\quad 0.715$ Ref.

Figure 3

C-statistics for predicting postoperative AE-ILD Concordance index (C-index) for predicting postoperative acute exacerbation of interstitial lung disease (AE-ILD) was significantly improved when HMGB1 and operative time were incorporated into the model with covariates calculated by previously reported risk scoring system as follows: $5 \times$ (history of $\mathrm{AE}$ ) $+4 \times$ (surgical procedures) $+4 \times$ (usual interstitial pneumonia appearance in computed tomography scan) $+3 \times$ (male sex) $+3 \times$ (preoperative steroid use) $+2 \times($ serum Krebs von den Lungen- 6 level higher than $1000 \mathrm{U} / \mathrm{mL})+1 \times($ vital capacity lower than $80 \%)$.

\section{Supplementary Files}


This is a list of supplementary files associated with this preprint. Click to download.

- Supplementarylnformation.pdf 